\title{
Generalized Guidance Law for Collision Courses
}

\author{
Yoriaki Baba* and Makoto Yamaguchi $\dagger$ \\ National Defense Academy, Yokosuka 239, Japan \\ and \\ Robert M. Howe \\ University of Michigan, Ann Arbor, Michigan 48109
}

\begin{abstract}
A new generalized guidance law for collision courses is presented. When the missile and target axial accelerations or decelerations are constant, there exists a rectilinear collision course. The guidance law presented, which is called the true guidance law, gives theoretical lateral acceleration commands to guide the missile on a collision course. However, since it is very difficult to realize the true guidance law on most existing tactical missiles, this paper shows a method for simply implementing the guidance law, which is called the simplified guidance law. The small perturbation equation of the true guidance law shows that the definition of an effective navigation constant is the same expression as that in the case of conventional proportional navigation. The performance of the two guidance laws presented is compared with that of proportional navigation using simulation studies of a simple model of a short range air-to-air missile. The simulation results show that the guidance laws presented can intercept the target using far smaller lateral acceleration commands than prepared for proportional navigation. The inner launch envelope shows that the guidance laws presented provide an overall performance improvement over proportional navigation.
\end{abstract}

\section{Nomenclature}

$a_{m} \quad=$ missile axial acceleration vector, $a_{m}=\left|a_{m}\right|$

$a_{t} \quad=$ target axial acceleration vector, $a_{t}=\left|a_{t}\right|$

$\boldsymbol{e}_{1}, \boldsymbol{e}_{2}=$ unit vectors

$F \quad=$ desired acceleration command vector

$M D=$ miss distance

$N \quad=$ navigation constant

$N_{e} \quad=$ effective navigation constant

$n_{\max }=$ maximum lateral load factor

$\boldsymbol{R} \quad=$ relative distance vector $\left(=\boldsymbol{r}_{t}-\boldsymbol{r}_{m}\right), \boldsymbol{R}=|\boldsymbol{R}|$

$r_{m} \quad=$ missile position vector

$r_{m c}=$ correct missile position vector

$r_{t} \quad=$ target position vector

$s \quad=$ differential operator

$T_{m} \quad=$ missile time constant

$t_{f} \quad=$ total flight time

$t_{\text {go }} \quad=$ time-to-go

$V_{m} \quad=$ missile velocity vector, $V_{m}=\left|V_{m}\right|$

$V_{m c}=$ correct missile velocity vector, $V_{m}=\left|V_{m c}\right|$

$V_{t} \quad=$ target velocity vector, $V_{t}=\left|\boldsymbol{V}_{t}\right|$

$x, y=$ elements of $\Delta r_{m}$

$\Delta \boldsymbol{r}_{m}=\boldsymbol{r}_{m}-\boldsymbol{r}_{m c}$

$\Delta \boldsymbol{V}_{m}=\boldsymbol{V}_{m}-\boldsymbol{V}_{m c}$

$\theta \quad=$ missile flight-path angle

$\dot{\theta}=$ missile flight-path rate vector

$\mu \quad=\phi_{t}-\phi_{m}$

$\sigma \quad=$ LOS angle

$\dot{\sigma} \quad=$ LOS rate vector

$\phi_{m} \quad=$ missile flight-path angle to LOS

$\phi_{t} \quad=$ target flight-path angle to LOS
Received July 18, 1991; presented as Paper 91-2782 at the AIAA Guidance, Navigation, and Control Conference, New Orleans, LA, Aug. 12-14, 1991; revision received July 16, 1992; accepted for publication Aug. 4, 1992. Copyright (C) 1991 by the American Institute of Aeronautics and Astronautics, Inc. All rights reserved.

*Associate Professor, Department of Aerospace Engineering. Member AIAA.

$\dagger$ Post-Graduate Student, Department of Aerospace Engineering. $\$$ Professor, Department of Aerospace Engineering. Member AIAA.

\section{Introduction}

$\mathrm{T}$ is well known that a conventional proportional navigation $(\mathrm{PN})$ is an adequate missile guidance law when the missile and target velocities remain constant. ${ }^{1-3}$ In practice, however, the missile and target velocities may change significantly. For instance, a short range air-to-air missile (AAM) has nearly constant axial acceleration during the boost phase. Also, the axial deceleration of a surface-to-air missile due to air drag after thrust cutoff can be assumed nearly constant. On the other hand, a target may have constant axial acceleration or slowdown. These kinds of axial accelerations or decelerations may seriously influence the performance of the missile guided by PN. Chadwick developed the approximate analytical solution for the miss distance of a proportional navigation missile with axial slowdown after sustainer motor cutoff, but he mentioned nothing about a guidance law. ${ }^{4}$

When the missile and target accelerations or decelerations are constant, there exists the rectilinear collision course. Then, if we know the magnitudes of these accelerations or decelerations and the initial time-to-go, the missile can be guided to a collision course. A missile flying on this collision course does not require further acceleration commands to hit the target. For example, Fig. 1 compares the rectilinear collision course with the trajectory achieved with PN. Since PN does not take into account changes in velocity, the trajectory with PN is curved, as shown in Fig. 1. In other words, the missile guided by $\mathrm{PN}$ requires more acceleration commands as it gets close to the target.

First, this paper presents the generalized guidance law for a missile with constant axial acceleration against a target with constant acceleration. Next, the small perturbation equation of the guidance law is derived. This shows that the definition of an effective navigation constant reduces to the same expression as that in the case of PN. An appropriate effective navigation constant can then be determined by integrating the small perturbation equation. Though the generalized guidance law gives the theoretical acceleration to guide a missile on a collision course, it is very difficult to implement this guidance law on most existing tactical missiles. Therefore, this paper shows a simplified method for implementing the guidance law. Finally, the performance of the guidance laws presented is compared with that of $\mathrm{PN}$ using simulation studies of the simple AAM models. 


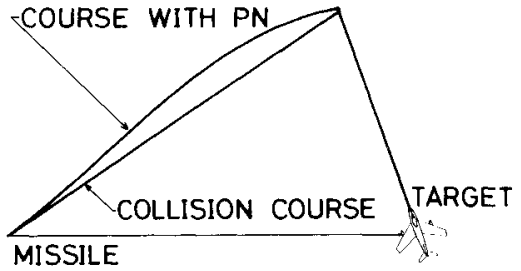

Fig. 1 Rectilinear collision course and the course achieved with PN.

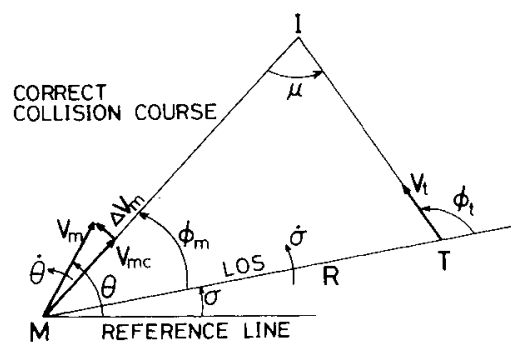

Fig. 2 Intercept geometry.

\section{Derivation of a Generalized Guidance Law}

Figure 2 shows the intercept geometry of a missile intercepting a target. $M$ and $T$ represent the actual positions of a missile and a target, respectively, at time $t$. From Fig. 2, the line-ofsight (LOS) rate is given by the following vector equation:

$$
\dot{\boldsymbol{\sigma}}=\frac{\boldsymbol{R} \times\left(\boldsymbol{V}_{t}-\boldsymbol{V}_{m}\right)}{R^{2}}
$$

Assuming that the missile is flying at constant acceleration $a_{m}$ and the target is flying at constant acceleration $a_{t}$, let the triangle $I T M$ be a collision triangle-that is, $I$ indicates the point of impact. $V_{m c}$ is the correct missile velocity vector to obtain a collision at $I$ and $\Delta \boldsymbol{V}_{m}$ is the deviation of missile velocity vector from $\boldsymbol{V}_{m c}$. Then we have

$$
\boldsymbol{V}_{m}=\boldsymbol{V}_{m c}+\Delta \boldsymbol{V}_{m}
$$

Substituting Eq. (2) into Eq. (1), we obtain

$$
\dot{\boldsymbol{\sigma}}=\frac{\boldsymbol{R} \times\left(\boldsymbol{V}_{t}-\boldsymbol{V}_{m c}\right)}{R^{2}}+\frac{\boldsymbol{R} \times\left(-\Delta \boldsymbol{V}_{m}\right)}{R^{2}}
$$

The first term of the right side of Eq. (3) represents the correct LOS rate when the missile flies along the collision course. The second term is the deviation of the LOS rate from the correct one. If a missile is guided with a flight-path rate in proportion to the deviation of the LOS rate, assuming no missile dynamic lags, the missile flight-path rate becomes

$$
\dot{\theta}=N \frac{\boldsymbol{R} \times\left(-\Delta \boldsymbol{V}_{m}\right)}{R^{2}}
$$

where $N$ is the navigation constant. Then, the required lateral acceleration command for a missile to fly along the correct collision course is given by

$$
\begin{aligned}
\boldsymbol{F} & =\dot{\boldsymbol{\theta}} \times \boldsymbol{V}_{m} \\
& =-N \frac{\left\{\left(V_{m c}-\boldsymbol{V}_{m}\right) \times \boldsymbol{R}\right\} \times \boldsymbol{V}_{\mathrm{m}}}{R^{2}}
\end{aligned}
$$

From Fig. 2, $V_{m c}$ can be written as

$$
\boldsymbol{V}_{m c}=V_{m}\left(\frac{\sin \phi_{m} \boldsymbol{V}_{t}}{\sin \phi_{t} V_{t}}+\frac{\sin \mu \boldsymbol{R}}{\sin \phi_{t} R}\right)
$$

Substituting Eq. (6) into Eq. (5), we obtain

$$
\boldsymbol{F}=\frac{N}{R^{2}}\left\{\left(V_{m}-\frac{V_{m} \sin \phi_{m}}{V_{t} \sin \phi_{t}} \boldsymbol{V}_{t}\right) \times \boldsymbol{R}\right\} \times \boldsymbol{V}_{m}
$$

If we let $t_{\text {go }}$ be the time-to-go, from Fig. 2, we have

$$
\boldsymbol{V}_{m c} t_{\mathrm{go}}+\frac{\boldsymbol{a}_{m}}{2} t_{\mathrm{go}}^{2}=\boldsymbol{R}+\boldsymbol{V}_{t} t_{\mathrm{go}}+\frac{\boldsymbol{a}_{t}}{2} t_{\mathrm{go}}^{2}
$$

From Fig. 2, the component of Eq. (8) perpendicular to $R$ is given by

$$
\left(V_{m} t_{\mathrm{go}}+\frac{a_{m}}{2} t_{\mathrm{go}}^{2}\right) \sin \phi_{m}=\left(V_{t} t_{\mathrm{go}}+\frac{a_{t}}{2} t_{\mathrm{go}}^{2}\right) \sin \phi_{t}
$$

Dividing Eq. (9) by $t_{\mathrm{go}} \sin \phi_{t}$, we obtain

$$
V_{m}\left(1+\frac{a_{m} t_{\mathrm{go}}}{2 V_{m}}\right) \frac{\sin \phi_{m}}{\sin \phi_{t}}=V_{t}\left(1+\frac{a_{t} t_{\mathrm{go}}}{2 V_{t}}\right)
$$

Equation (10) can be rewritten as

$$
\frac{V_{m} \sin \phi_{m}}{V_{t} \sin \phi_{t}}=\frac{1+\epsilon_{t}}{1+\epsilon_{m}}
$$

where

$$
\begin{gathered}
\epsilon_{m}=\frac{a_{m} t_{\mathrm{go}}}{2 V_{m}} \\
\epsilon_{t}=\frac{a_{t} t_{\mathrm{go}}}{2 V_{t}}
\end{gathered}
$$

Substituting Eq. (11) into Eq. (7), we obtain

$$
\boldsymbol{F}=N / R^{2}\left[\left(V_{m}-k V_{t}\right) \times R\right] \times V_{m}
$$

where

$$
k=\frac{1+\epsilon_{t}}{1+\epsilon_{m}}
$$

This is the generalized guidance law for a missile with constant axial acceleration to intercept a target with constant axial acceleration. If both a missile and a target have no acceleration, that is, constant velocity, $\epsilon_{m}$ and $\epsilon_{t}$ are zero and Eq. (14a) reduces to a conventional proportional navigation guidance law. Since the value of $t_{\mathrm{go}}$ is required in order to compute $\epsilon_{m}$ and $\epsilon_{t}$, we derive the equation for $t_{\text {go }}$. From Fig. 2, we have

$$
\begin{gathered}
\left(V_{m}+\frac{a_{m} t_{\mathrm{go}}}{2}\right) t_{\mathrm{go}} \cos \mu=R \cos \phi_{t}+V_{t} t_{\mathrm{go}}+\frac{a_{t}}{2} t_{\mathrm{go}}^{2} \\
\left(V_{m}+\frac{a_{m} t_{\mathrm{go}}}{2}\right) t_{\mathrm{go}} \sin \mu=R \sin \phi_{t}
\end{gathered}
$$

Squaring both sides of Eqs. (15) and (16) and adding them, we obtain

$$
\begin{aligned}
& \left(\frac{a_{t}^{2}-a_{m}^{2}}{4}\right) t_{\mathrm{go}}^{4}+\left(V_{t} a_{t}-V_{m} a_{m}\right) t_{\mathrm{go}}^{3}+\left(V_{t}^{2}-V_{m}^{2}\right) t_{\mathrm{go}}^{2} \\
& +2 R\left(V_{t} t_{\mathrm{go}}+\frac{a_{t}}{2} t_{\mathrm{go}}^{2}\right) \cos \phi_{t}+R^{2}=0
\end{aligned}
$$

Assuming that $V_{m}, V_{t}, a_{m}, a_{t}, R$, and $\phi_{t}$ are known, $t_{\mathrm{go}}$ can be computed from Eq. (17); $\epsilon_{m}$ and $\epsilon_{1}$ can be obtained from Eqs. (12) and (13); and the required guidance acceleration commands are then computed from Eq. (14). We call this guidance law the true guidance law. 


\section{Small Perturbation Equation}

Equation (14) gives the generalized guidance law for collision courses where time-to-go is computed from Eq. (17). Since this equation is complicated, however, it is difficult to select the value of the navigation constant $N$. To choose $N$, it is useful to linearize Eq. (14a) about a correct collision course. For simplicity, we will consider the problem in two dimensions. Figure 3 shows the engagement model for linearization, where the target is flying along the reference trajectory. In Fig. $3, O$ is the origin of the inertial frame, $M_{0}$ indicates the position that a missile would have had at the time $t$ in the correct trajectory case and $M$ displays the actual position of a missile at time $t$. The $\phi_{m c}, \phi_{t c}$ and $\mu_{c}$ are the correct angles such that $M_{0} I$ and $T I$ become the correct collision courses for missile and a target, respectively. From Fig. 3, we have

$$
\begin{gathered}
\boldsymbol{r}_{m}=\boldsymbol{r}_{m c}+\Delta \boldsymbol{r}_{m} \\
\boldsymbol{V}_{m}=\boldsymbol{V}_{m c}+\Delta \boldsymbol{V}_{m} \\
\Delta \boldsymbol{r}_{m}=x \boldsymbol{e}_{1}+y \boldsymbol{e}_{2} \\
\Delta \boldsymbol{V}_{m}=\dot{x} \boldsymbol{e}_{1}+\dot{y} \boldsymbol{e}_{2} \\
\boldsymbol{R}=\boldsymbol{r}_{t}-\boldsymbol{r}_{m c}-\Delta \boldsymbol{r}_{m} \\
\boldsymbol{R}_{c}=\boldsymbol{r}_{t}-\boldsymbol{r}_{m c}
\end{gathered}
$$

Substituting Eqs. (18), (19), (22), (23) into the numerator of Eq. (14a), neglecting terms higher than second order in $\Delta \boldsymbol{r}_{m}$ and $\Delta \boldsymbol{V}_{m}$, and using Eqs. (20) and (21) we obtain

$$
\begin{aligned}
\boldsymbol{F} & =\dot{s}\left(N V_{m} / R^{2}\right)\left(-\dot{x} R \sin \phi_{m c}-x k V_{t} \sin \mu_{c}\right. \\
& \left.-\dot{y} R \cos \phi_{m c}+y k V_{t} \cos \mu_{c}-V_{m} y\right) e_{2}
\end{aligned}
$$

Since the external force on the missile is not applied in the direction of $e_{1}$, except for the constant acceleration $a_{m}$, we have

$$
\ddot{x}(t)=0
$$

Assuming $x(0)=\dot{x}(0)=0$, we obtain

$$
x(t)=0
$$

On the other hand, we have

$$
\boldsymbol{F}=\ddot{y} \boldsymbol{e}_{2}
$$

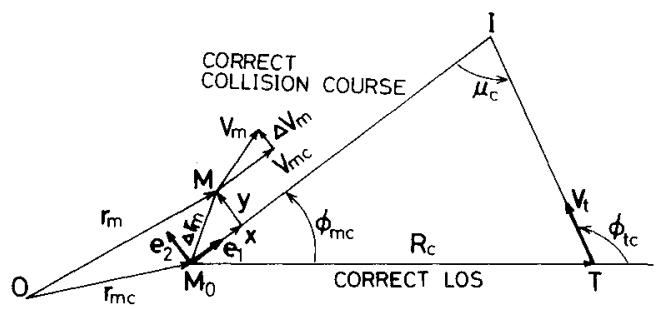

Fig. 3 Engagement model for linearization.

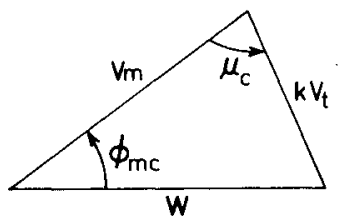

Fig. 4 Definition of $W$.
Substituting Eqs. (26) and (27) into Eq. (24), we have

$$
\ddot{y}=-\frac{N V_{m} \cos \phi_{m c}}{R} \dot{y}+\frac{N V_{m}}{R^{2}}\left(-V_{m}+k V_{t} \cos \mu_{c}\right) y
$$

Let us define $V_{c}$ and $W$ as follows:

$$
\begin{gathered}
V_{c}=V_{m} \cos \phi_{m c}-V_{t} \cos \phi_{t c} \\
W=\left(k^{2} V_{t}^{2}+V_{m}^{2}-2 k V_{t} V_{m} \cos \mu_{c}\right)^{0.5}
\end{gathered}
$$

$V_{c}$ becomes the closing velocity, and $W$ is the length of the side of the triangle, as shown in Fig. 4. From Fig. 4, we obtain

$$
V_{m}-k V_{t} \cos \mu_{c}=W \cos \phi_{m c}
$$

From Eqs. (29) and (31), Eq. (28) can be written as

$$
\begin{aligned}
\ddot{y} & =-\frac{N V_{m} \cos \phi_{m c}}{R}\left(\dot{y}+\frac{W}{R} y\right) \\
& =-N_{e} \frac{V_{c}}{R}\left(\dot{y}+\frac{W}{R} y\right)
\end{aligned}
$$

where the parameter $N_{e}$ is defined by

$$
N_{e}=\frac{N V_{m} \cos \phi_{m c}}{V_{c}}
$$

As is obvious from Eq. (33), $N_{e}$ is the same expression as the effective navigation constant in the case of PN. Thus we also call $N_{e}$ an effective navigation constant in this paper. Equation (32) is the small perturbation equation for Eq. (14a). The initial conditions for a launch error are given by

$$
y(0)=0, \quad \dot{y}(0)=V_{m}(0) \gamma_{0}
$$

and the miss distance is given by

$$
M D=y\left(t_{f}\right)
$$

As mentioned earlier, we have assumed until now that there are no missile dynamic lags. However, if we assume that the missile dynamics can be represented as a linear system with transfer operator $Y(s)$, where $s$ is the operator $\mathrm{d} / \mathrm{d} t$ and $Y(s)$ is the ratio of two polynomials in $s$, then the perturbation equation for the generalized guidance law becomes, instead of Eq. (32),

$$
\ddot{y}=-N_{e} Y(s) \frac{V_{c}}{R}\left(\dot{y}+\frac{W}{R} y\right)
$$

As a specific example, consider the case of a missile with a simple time lag $T_{m}$. Thus, let

$$
Y(s)=\frac{1}{1+T_{m} s}
$$

Substituting Eq. (37) into Eq. (36), we obtain

$$
T_{m} y^{\prime \prime \prime}+\ddot{y}=-N_{e} \frac{V_{c}}{R}\left(\dot{y}+\frac{W}{R} y\right)
$$

Let us consider solving Eq. (38) numerically for a launch error $\gamma_{0}$. From Eq. (34), the initial values become

$$
y(0)=0, \quad \dot{y}(0)=V_{m}(0) \gamma_{0}, \quad \ddot{y}(0)=0
$$

To solve Eq. (38), we assume $t_{\mathrm{go}}$ is given by

$$
t_{\mathrm{go}}=t_{f}-t
$$


where $t_{f}$ is the total flight time. $V_{m}$ and $V_{t}$ are given by

$$
\begin{gathered}
V_{m}=V_{m 0}+a_{m} t \\
V_{t}=V_{t 0}+a_{t} t
\end{gathered}
$$

where $V_{m 0}$ and $V_{t 0}$ are the initial values of $V_{m}$ and $V_{t}$. Letting $V_{m a}$ and $V_{t a}$ be the average velocities of the missile and target, respectively, we have

$$
\begin{gathered}
V_{m a}=V_{m 0}+\left(a_{m} t_{f} / 2\right) \\
V_{t a}=V_{t 0}+\left(a_{t} t_{f} / 2\right)
\end{gathered}
$$

Then, the average closing velocity $V_{c a}$ becomes

$$
V_{c a}=V_{m a} \cos \phi_{m c}-V_{t a} \cos \phi_{t c}
$$

The relative distance is given by

$$
R=V_{c a} t_{f}-\left\{\left(V_{m 0}+\frac{a_{m}}{2} t\right) \cos \phi_{m c}-\left(V_{t 0}+\frac{a_{t}}{2} t\right) \cos \phi_{t c}\right\} t
$$

If $V_{m 0}, V_{t 0}, a_{m}, a_{t}, \mu_{c}$, and $t_{f}$ are given, $\phi_{m c}$ is computed from the following equation:

$$
\phi_{m c}=\sin ^{-1}\left\{\frac{\sin \mu_{c}\left(V_{t o} t_{f}+a_{t} t_{f}^{2} / 2\right)}{h}\right\}
$$

where

$$
\begin{aligned}
h & =\left\{\left(V_{t 0} t_{f}+\frac{a_{t}}{2} t_{f}^{2}\right)^{2}+\left(V_{m 0} t_{f}+\frac{a_{m}}{2} t_{f}^{2}\right)^{2}\right. \\
& \left.-2\left(V_{t 0} t_{f}+\frac{a_{t}}{2} t_{f}^{2}\right)\left(V_{m 0} t_{f}+\frac{a_{m}}{2} t_{f}^{2}\right) \cos \mu_{c}\right\}^{0.5}
\end{aligned}
$$

Equation (38) was numerically integrated for the case where $V_{m 0}=288 \mathrm{~m} / \mathrm{s}, \quad V_{t 0}=288 \mathrm{~m} / \mathrm{s}, \quad a_{m}=154 \mathrm{~m} / \mathrm{s}^{2}, \quad a_{t}=0$, $T_{m}=0.4 \mathrm{~s}$, and $\mu_{\mathrm{c}}=90 \mathrm{deg}$. Selecting values of $N_{e}=3,4,5$, and 6 , analysis of the dimensionless miss distance $y\left(t_{f}\right) /$ $V_{m a} \gamma_{0} T_{m}$ vs dimensionless time of flight $t_{f} / T_{m}$, the deviation histories from the correct position, and the lateral acceleration command histories in normalized form, where $t_{f}$ was set equal to $4 \mathrm{~s}$, showed that for this example a reasonable value of $N_{e}$ would be in the range of $4-5$.

\section{Simplified Construction of the Guidance Law}

To construct the true guidance law given by Eq. (14), we must measure or estimate $V_{m}, V_{t}, a_{m}, a_{t}, R$, and $\phi_{t}$ and compute $t_{\text {go }}$ from Eq. (17) in real time. In general, however, most present tactical missiles do not carry the kind of instruments needed to measure all of these variables. Thus it becomes very difficult to realize Eq. (14). In this section, let us consider the real mechanization of Eq. (14). The term $V_{m}-k V_{t}$ in Eq. (14a) can be rewritten as

$$
\begin{aligned}
\boldsymbol{V}_{m}-k \boldsymbol{V}_{t} & =\boldsymbol{V}_{m}-\frac{1+\epsilon_{t}}{1+\epsilon_{m}} \boldsymbol{V}_{t} \\
& =\left(\frac{1+\epsilon_{t}}{1+\epsilon_{m}}\right)\left(\boldsymbol{V}_{m}-\boldsymbol{V}_{t}\right)+\left(\frac{\epsilon_{m}-\epsilon_{t}}{1+\epsilon_{m}}\right) V_{m}
\end{aligned}
$$

Substituting Eq. (49) into Eq. (14a), we obtain

$$
\begin{aligned}
\boldsymbol{F} & =\frac{N\left(1+\epsilon_{t}\right)}{\left(1+\epsilon_{m}\right)} \frac{\left\{\left(\boldsymbol{V}_{m}-\boldsymbol{V}_{t}\right) \times \boldsymbol{R}\right\} \times \boldsymbol{V}_{m}}{R^{2}} \\
& +\frac{N\left(\epsilon_{m}-\epsilon_{t}\right)}{\left(1+\epsilon_{m}\right)} \frac{\left(\boldsymbol{V}_{m} \times \boldsymbol{R}\right) \times \boldsymbol{V}_{m}}{R^{2}}
\end{aligned}
$$

The first term represents proportional navigation with the navigation constant $N\left(1+\epsilon_{t}\right) /\left(1+\epsilon_{m}\right)$ and the second term represents pure pursuit navigation with the navigation constant $N\left(\epsilon_{m}-\epsilon_{t}\right) /\left(1+\epsilon_{m}\right)$. We need the values of $\epsilon_{m}$ and $\epsilon_{t}$ to determine the two navigation constants. Though $V_{m}, V_{t}, a_{m}$, $a_{t}, R$, and $\phi_{t}$ must be measured to compute $\epsilon_{m}$ and $\epsilon_{t}$ precisely, we do not necessarily need the correct values of $\epsilon_{m}$ and $\epsilon_{t}$. This is because, even if the navigation constants change somewhat from the optimal values, the performances of the guidance law will not be affected significantly. Therefore, if $t_{f}, a_{m}, a_{t}, V_{m 0}$, and $V_{t 0}$ are given from the launcher or the parent aircraft before launch, we can estimate the values of $t_{\mathrm{go}}, V_{m}$, and $V_{t}$ as follows:

$$
\begin{gathered}
t_{\mathrm{go}}=t_{f}-t \\
V_{m}=V_{m 0}+a_{m} t \\
V_{t}=V_{t 0}+a_{t} t
\end{gathered}
$$

Using these values, we can compute $\epsilon_{m}$ and $\epsilon_{t}$ from Eqs. (12) and (13) and simply mechanize Eq. (50). The block diagram representation of Eq. (50) is depicted in Fig. 5. Here, the effective navigation constant $N_{e}$ defined by Eq. (33) is used instead of $N$. We call this implementation the simplified guidance law. In Fig. $5, K_{p}$ represents the gain for pure pursuit guidance and is chosen so that the trajectory achieved with the simplified guidance law approaches that with the true guidance law as nearly as possible. In other words, if $\mathrm{d}(t)$ represents the relative distance between the two trajectories, $K_{p}$ is chosen to minimize the following $P I$ :

$$
P I=\int_{0}^{t_{f}} \mathrm{~d}(t) \mathrm{d} t
$$

\section{Application to a Short Range AAM}

Let us apply the true guidance law and the simplified guidance law to the simple model of a short range air-to-air missile

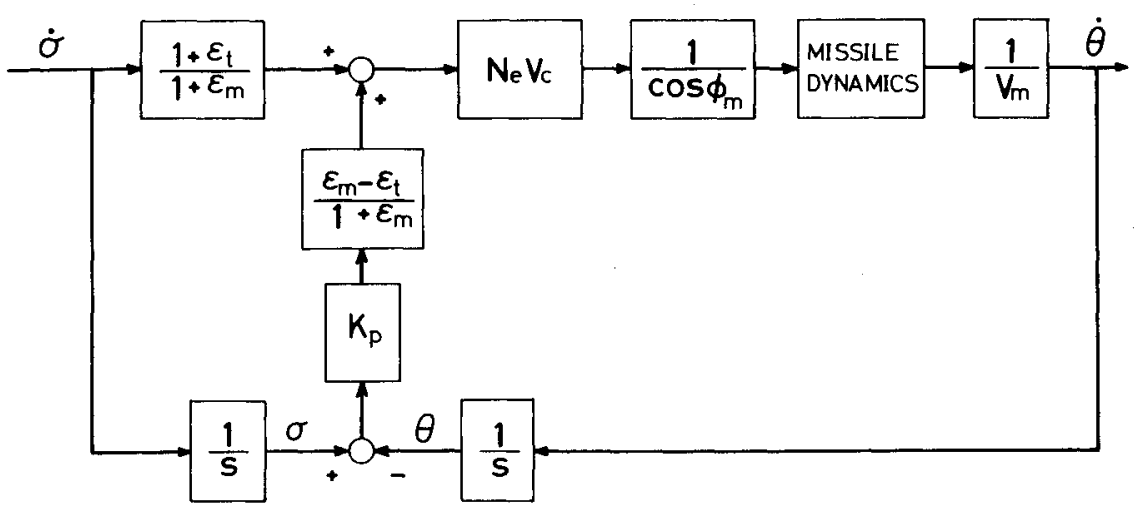

Fig. 5 Block diagram for the simplified guidance law. 
(SRAAM) and compare the results with that achieved with PN. Since most SRAAM have solid rocket motors, their axial accelerations can be assumed nearly constant during boost phase. On the other hand, the target velocity can be considered nearly constant because it is very difficult to change significantly the target velocity during the short intercept time. Let us assume that both the missile and the target are particles; their trajectories are limited to two dimensions; and the total dynamics of the guidance system, including the missile dynamics, a noise filter, etc., is given by a first-order lag with time constant $0.4 \mathrm{~s}$. The velocity of the target is constant at 288 $\mathrm{m} / \mathrm{s}$. The initial velocity of the missile is $288 \mathrm{~m} / \mathrm{s}$, and the acceleration is $154 \mathrm{~m} / \mathrm{s}^{2}$. Then, based on our previous discussion, we set $N_{e}=4.5$. Figures 6 and 7 are the graphs used to decide $K_{p}$. Figure 6 shows $P I$ vs $K_{p}$, where $\mu=90 \mathrm{deg}$ and $t_{f}=4 \mathrm{~s}$. From this figure, the optimal $K_{p}$ is 0.2 for $\mu=90 \mathrm{deg}$. Figure 7 displays the optimal $K_{p}$ vs $\mu$. Though the optimal value of $K_{p}$ depends on $\mu$ as shown in Fig. 7, we set $K_{p}=0.2$ because a broadside attack is the most severe case for a missile. $K_{p}$ is also a function of $t_{f}$; however, simulation results have shown that the optimal value of $K_{p}$ does not depend very much on $t_{f}$. Figure 8 displays one of the simulation results where the target is flying straight and the missile is launched along the collision course. The figure shows that the missile guided by the true guidance law flies straight without accelera-

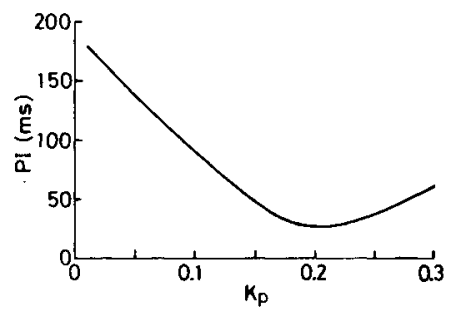

Fig. $6 P I$ vs $K_{p}$.

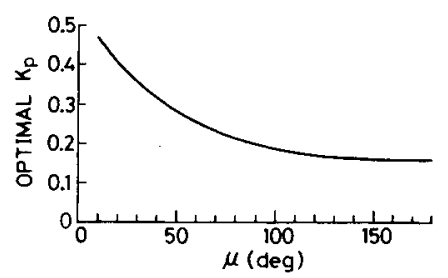

Fig. 7 Optimal $K_{p}$ as a function of $\mu$.

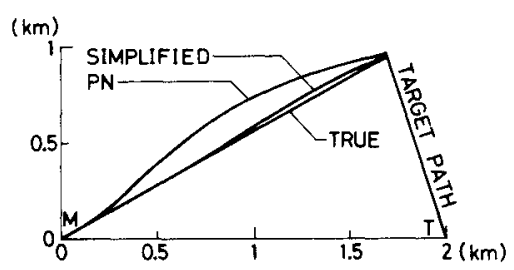

(a)

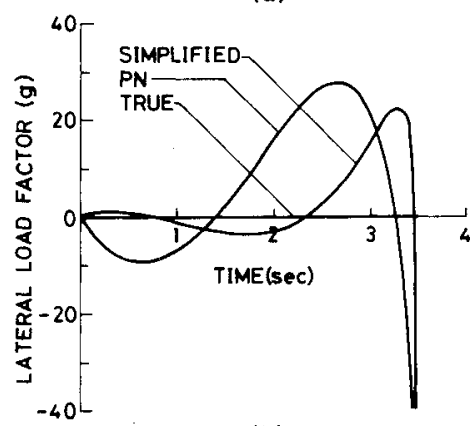

(b)

Fig. 8 Missile and target trajectories and lateral load factor time histories.

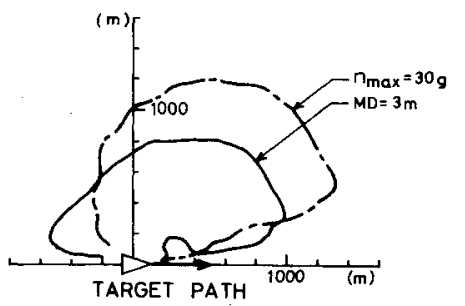

Fig. 9 Launch boundaries for miss and maximum lateral load factor to be $3 \mathrm{~m}$ and $30 \mathrm{~g}$, respectively: true guidance law.

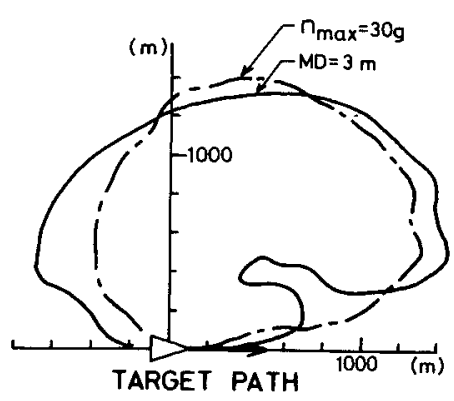

Fig. 10 Launch boundaries for miss and maximum lateral load factor to be $3 \mathrm{~m}$ and $30 \mathrm{~g}$, respectively: simplified guidance law.

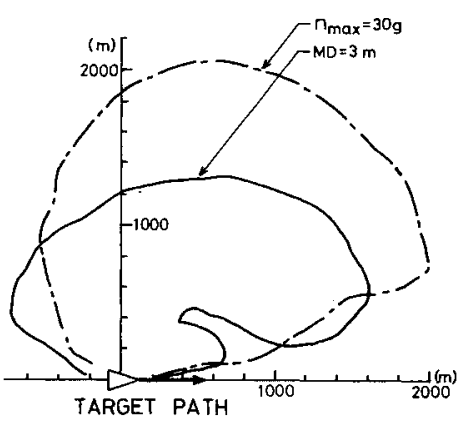

Fig. 11 Launch boundaries for miss and maximum lateral load factor to be $3 \mathrm{~m}$ and $30 \mathrm{~g}$, respectively: proportional navigation.

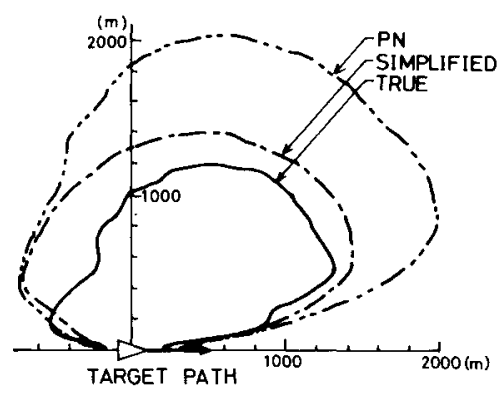

Fig. 12 Inner launch envelopes of AAM.

tion commands. The trajectory achieved with $\mathrm{PN}$ is curved and requires large acceleration commands. The effective navigation constant for PN was set equal to 4.5. The trajectory achieved with the simplified guidance law is also curved, but the deviation from the ideal trajectory is much smaller than the deviation with PN. Also, the required acceleration commands are smaller. Figures 9-11 show the launch boundaries for the miss distance and the maximum lateral load factor specified as $3 \mathrm{~m}$ and $30 \mathrm{~g}$, respectively. Figure 9 is based on the true guidance law, Fig. 10 is with the simplified guidance law, and Fig. 11 is with PN. Here it is assumed that the target is flying straight and the missile is launched against the target without lead angle; other conditions are the same as those described before. The inner launch envelope for SRAAM must simultaneously satisfy at least these two boundaries. 
Figure 12 compares the three inner launch envelopes achieved with the different guidance laws. Figures 11 and 12 show that the envelope with PN mainly depends on the boundary of the maximum load factor.

\section{Conclusion}

A new generalized guidance law for collision courses has been presented. When the missile and target axial accelerations or decelerations are constant, there exists a rectilinear collision course. The guidance law presented, which is called the true guidance law, gives the theoretical guidance acceleration commands to guide a missile on the collision course. The implementation of the true guidance law requires values of missile velocity, missile axial acceleration, target velocity, target axial acceleration, the relative distance between the missile and the target, and the target flight-path angle to LOS. In general, however, most present tactical missiles do not carry instruments to measure all these variables. Thus, it is very difficult to realize the true guidance law. Therefore, this paper has presented a method for approximately implementing the true guidance law by use of only the target and missile initial velocities and accelerations, and the initial values of time-togo, which are given from a launcher or a parent aircraft at launch. This implementation is called the simplified guidance law.

The small perturbation equation of the true guidance law has shown that the definition of an effective navigation constant is the same as that in the case of proportional navigation. Also, the appropriate value of the navigation constant can be defined by integrating the small perturbation equation. The guidance laws presented as well as proportional navigation were applied to a simple model of a short range air-to-air missile with constant acceleration. From simulations, the inner launch envelopes were generated and the following results were obtained.

1) The missile guided by the true guidance law flies straight and hits the target without further acceleration commands, provided there is no initial heading error.

2) The trajectory achieved with proportional navigation is quite curved, and large acceleration commands are required near the trajectory end even if the missile is launched along a collision course.

3) The trajectory with the simplified guidance law is also curved, but the deviation from the true collision course is far smaller than that with proportional navigation. Also, the required acceleration commands are smaller.

4) The inner launch envelope has shown that the guidance laws presented provide an overall performance improvement over proportional navigation.

\section{References}

'Howe, R. M., "Guidance," System Engineering Handbook, edited by R. E. Machol, W. P. Tanner Jr., and S. N. Alexander, McGraw-Hill, New York, 1965, Chap. 19.

${ }^{2}$ Garnell, P., Guided Weapon Control Systems, Pergamon Press, Oxford, England, UK, 1980, pp. 198-241.

${ }^{3}$ Nesline, F. W., and Zarchan, P., "A New Look at Classical vs Modern Homing Missile Guidance," Journal of Guidance and Control, Vol. 4, No. 1, 1981, pp. 78-85.

${ }^{4}$ Chadwick, W. R., "Miss Distance of Proportional Navigation Missile with Varying Velocity," Journal of Guidance, Control, and Dynamics, Vol. 8, No. 5, 1985, pp. 662-666. 